Denis Kotarski

Petar Piljek

Hrvoje Brezak

Josip Kasać

https://doi.org/10.21278/TOF.42101

ISSN 1333-1124

eISSN 1849-1391

\title{
CHATTERING-FREE TRACKING CONTROL OF A FULLY ACTUATED MULTIROTOR WITH PASSIVELY TILTED ROTORS
}

\begin{abstract}
Summary
In this paper, a control allocation scheme is presented for a multirotor type of an Unmanned Aerial Vehicle (UAV). The control allocation scheme depends on the multirotor configuration and rotor system parameters, and it enables analysis of dynamics of different multirotor designs depending on the purpose and the task which the multirotor has to carry out. The analysis of force and moment distribution in space shows that the non-flat design with passively tilted rotors can overcome an inherent underactuated condition of flat multirotor configurations. By increasing the tilt angle, the multirotor is able to achieve full controllability over its six degrees of freedom (6 DOFs). A robust chattering-free sliding mode asymptotic tracking control design of a fully actuated multirotor is presented. The simulation results show satisfying tracking performance of the proposed controller.
\end{abstract}

Key words: $\quad$ control allocation scheme, tilt angle, fully actuated multirotor, robust tracking control, RISE control

\section{Introduction}

UAVs are expected to be used for a wide range of tasks and to operate in hazardous conditions. UAVs can be classified into five main categories: I) rotary-wing; II) fixed-wing; III) flapping-wing; IV) blimp; and V) hybrid UAVs. Rotary-wing UAVs have either variable pitch propellers (helicopters) or fixed pitch propellers (multirotors). Because of their unique ability to carry out vertical take-off and landing (VTOL) together with stationary and low speed flight, multirotor UAVs are suitable for carrying out a variety of tasks.

Multirotor is a highly nonlinear, multivariable and inherently instable 6-DOF rigid body. Challenges facing this type of UAV and research areas are described in [1]. Multirotor UAVs can be classified as: a) flat (quadrotor, hexarotor, octarotor); b) coaxial flat (Y6, X8 multirotor); c) overlapping flat [3]; d) non-flat (passively or actively tilted) multirotor UAVs and e) their combinations.

Development and design of multirotors are considerably constrained by their size, weight and power consumption [2]. There are a few papers [3, 4] that deal with configuration parameters important for the design of multirotors intended to perform different tasks such as disaster site observations or search and rescue missions $[5,6]$. 
Several groups of researchers deal with novel configurations in order to change various multirotor properties [7, 8]. A novel overactuated quadrotor UAV based on actively tilted rotors proposed in [9] is able to overcome underactuation limitation. However, it requires additional motors, which makes the system more complex and also increases power consumption. Therefore, passively tilted non-flat configuration is more suitable for analysis and realization. Furthermore, an analysis of the tilt angle parameter is given in [10]. The drawback of the presented papers is a limitation in the mathematical representation of the multirotor configuration, so the analysis is also restricted to specific multirotor parameters.

In this paper, a full nonlinear mathematical model of a multirotor UAV is described. It is divided into rigid body dynamics and control allocation scheme. The control allocation scheme describes a mapping of the rotor angular velocities to a control vector. Open loop simulations enable analysis of the dynamics and energy consumption of different multirotor configurations and rotor system physical parameters. The analysis of dynamics facilitates a design of diverse multirotor configurations for various purposes. Force and moment distribution in space show that the non-flat design with passively tilted rotors can overcome the inherent underactuated property of flat multirotor configurations, hence the multirotor is able to achieve full controllability and can decouple position from orientation. This fact has a significant influence on the multirotor controller design. The non-flat configuration provides six independent control variables, one for each degree of freedom, contrary to the flat configuration, which provides only four independent control variables. Such a decoupling control system design provides a more efficient realization of different control objectives, such as rejection of external disturbances.

During outdoor flights, aircraft are very often struggling with wind gusts or other environmental disturbances. Robust disturbance rejection control is presented in $[11,12]$ including an experimental validation on a quadrotor test bed. A quadrotor robust tracking controller is proposed in [13] with respect to the unmodelled dynamics which provides rejection of disturbances, for the purpose of solving the trajectory tracking problem. In [14], RISE (Robust Integral of the Sign of the Error) tracking control is presented.

The proposed control design is based on a fully actuated multirotor. The resulting controller is based on sliding mode control laws for attitude and position control defined by using the Lyapunov stability analysis proposed in [13]. The controller provides asymptotic tracking in the presence of external forces, such as wind gusts. Although the controller enables exponential tracking, chattering of control variables occurs. Therefore, the RISE type of control is proposed to overcome the chattering problem while preserving asymptotic tracking properties of the sliding mode controller.

The paper is organized as follows. In section 2, a mathematical model of the multirotor is derived. Based on the tilt angle, a force moment mapping analysis is provided in section 3. A design of a robust tracking control for a fully actuated multirotor is presented in section 4 . Simulation results are presented in section 5. Conclusion and future work is discussed in section 6.

\section{Multirotor mathematical model}

The mathematical model describes multirotor dynamics and behaviour with respect to the input values of the model and external influences on the multirotor. It can be considered as a function that is mapping inputs to outputs. By using a mathematical model, it is possible to predict position and orientation of a multirotor by knowing angular velocities of propellers, i.e. the model enables computer simulation of multirotor behaviour in different conditions. 


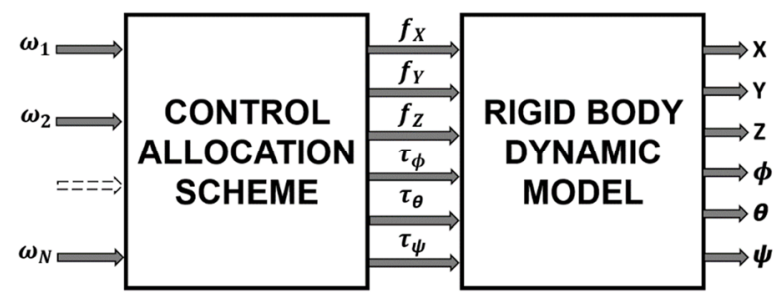

Fig. 1 Multirotor mathematical model

It is necessary to define two Cartesian coordinate systems: the Earth fixed frame (Eframe, $\mathcal{F}^{E}$ ) and the body fixed frame $\left(\mathrm{B}\right.$-frame, $\mathcal{F}^{B}$ ). Some multirotor physical properties are measured in $\mathcal{F}^{E}$, while some properties are measured in $\mathcal{F}^{B}$.

$\mathcal{F}^{E}$ is the inertial right-handed coordinate system where the positive direction of the $Z$ axis is in the direction normal to the Earth ground level. The multirotor position $\xi=$ $\left[\begin{array}{lll}X & Y & Z\end{array}\right]^{\mathrm{T}}$ and orientation $\boldsymbol{\eta}=\left[\begin{array}{lll}\phi & \theta & \psi\end{array}\right]^{\mathrm{T}}$ are defined in $\mathcal{F}^{E}$.

$\mathcal{F}^{B}$ is fixed on the multirotor body and it is also a right-handed coordinate system. It is assumed that the origin of $\mathcal{F}^{B}$ coincides with the multirotor centre of gravity (COG) and that the principal inertia axes of the multirotor body coincide with the $\mathcal{F}^{B}$ coordinate axes. The linear velocities $\mathbf{v}^{B}=\left[\begin{array}{lll}u & v & w\end{array}\right]^{\mathrm{T}}$, angular velocities $\boldsymbol{\omega}^{B}=\left[\begin{array}{lll}p & q & r\end{array}\right]^{\mathrm{T}}$, forces $\mathbf{F}=$ $\left[\begin{array}{lll}F_{X} & F_{Y} & F_{Z}\end{array}\right]^{\mathrm{T}}$ and moments $\mathbf{T}=\left[\begin{array}{lll}T_{\phi} & T_{\theta} & T_{\psi}\end{array}\right]^{\mathrm{T}}$ are defined in $\mathcal{F}^{B}$.

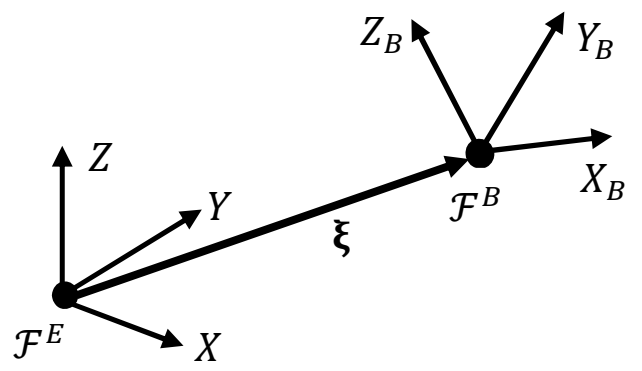

Fig. 2 Multirotor coordinate systems

Motion equations are more suitable for formulation with respect to $\mathcal{F}^{B}$ for several reasons: the system inertia matrix is time-invariant, simplified equations because of the multirotor frame symmetry, sensors measurements are easily converted to $\mathcal{F}^{B}$ and control variables equations simplification.

Kinematics of a rigid body with 6 DOFs is given by:

$$
\dot{\boldsymbol{\varepsilon}}=\boldsymbol{\Theta v}
$$

where $\dot{\boldsymbol{\varepsilon}}=\left[\begin{array}{ll}\dot{\boldsymbol{\xi}} & \dot{\boldsymbol{\eta}}\end{array}\right]^{\mathrm{T}}$ is the generalized velocity vector in $\mathcal{F}^{E}, \mathbf{v}=\left[\begin{array}{ll}\mathbf{v}^{B} & \boldsymbol{\omega}^{B}\end{array}\right]^{\mathrm{T}}$ is the generalized velocity vector in $\mathcal{F}^{B}$, and $\boldsymbol{\Theta}$ is the generalized rotation and transformation matrix.

$$
\boldsymbol{\Theta}=\left[\begin{array}{cc}
\mathbf{R} & \mathbf{0}_{3 \times 3} \\
\mathbf{0}_{3 \times 3} & \mathbf{\Omega}_{\boldsymbol{B}}
\end{array}\right]
$$

where $\mathbf{R}$ is the rotation matrix which maps the linear velocity vector from $\mathcal{F}^{B}$ to $\mathcal{F}^{E}$.

$$
\mathbf{R}=\left[\begin{array}{ccc}
c_{\psi} c_{\theta} & c_{\psi} s_{\theta} s_{\phi}-s_{\psi} c_{\phi} & c_{\psi} s_{\theta} c_{\phi}+s_{\psi} s_{\phi} \\
s_{\psi} c_{\theta} & s_{\psi} s_{\theta} s_{\phi}+c_{\psi} c_{\phi} & s_{\psi} s_{\theta} c_{\phi}-c_{\psi} s_{\phi} \\
-s_{\theta} & c_{\theta} s_{\phi} & c_{\theta} c_{\phi}
\end{array}\right]
$$

where $c_{i}=\cos (i), \mathrm{s}_{j}=\sin (j)$. 
Matrix $\boldsymbol{\Omega}_{B}$ is the transformation matrix that transfers angular velocities from $\mathcal{F}^{B}$ to $\mathcal{F}^{E}$ $[15]$.

$$
\boldsymbol{\Omega}_{B}=\left[\begin{array}{ccc}
1 & s_{\phi} t_{\theta} & c_{\phi} t_{\theta} \\
0 & c_{\phi} & -s_{\phi} \\
0 & s_{\phi} / c_{\theta} & c_{\phi} / c_{\theta}
\end{array}\right]
$$

where $t_{i}=\tan (i)$. Since the multirotor attitude is close to the hovering state, $\boldsymbol{\Omega}_{B}$ is close to the identity matrix, making the angular acceleration equations in $\mathcal{F}^{E}$ and $\mathcal{F}^{B}$ the same.

\subsection{Rigid body dynamic model}

Multirotor dynamics is described by differential equations that were derived by using the Newton-Euler method and are the same as those describing a rotating 6-DOF rigid body $[16,17]$. The mass $m$ and the inertia of the body $\mathbf{I}$ are taken into consideration. By applying the assumption that the multirotor frame has symmetrical structure, i.e. the principal inertia axes coincide with the $\mathcal{F}^{B}$ coordinate axes, the inertia matrix becomes the diagonal matrix $\mathbf{I}=\operatorname{diag}\left\{I_{X X}, I_{Y Y}, I_{Z Z}\right\}$.

The rigid body linear dynamics is described by

$$
m \dot{\mathbf{v}}^{B}+\boldsymbol{\omega}^{B} \times\left(m \mathbf{v}^{B}\right)=\mathbf{F}
$$

The angular dynamics is described by

$$
\mathbf{I} \dot{\boldsymbol{\omega}}^{B}+\boldsymbol{\omega}^{B} \times\left(\mathbf{I} \boldsymbol{\omega}^{B}\right)=\mathbf{T}
$$

where $\mathbf{F}$ is the force vector and $\mathbf{T}$ is the moment vector with respect to $\mathcal{F}^{B}$. The generalized force vector $\boldsymbol{\Lambda}=\left[\begin{array}{ll}\mathbf{F} & \mathbf{T}\end{array}\right]^{\mathrm{T}}$ can be divided into three components: the gravitational vector $\mathbf{g}_{\mathrm{B}}(\boldsymbol{\xi})$, the disturbance vector $\mathbf{d}=\left[\begin{array}{ll}\mathbf{d}_{f} & \mathbf{d}_{\tau}\end{array}\right]^{\mathrm{T}}$ and the control vector $\mathbf{u}_{B}=\left[\begin{array}{ll}\mathbf{f} & \boldsymbol{\tau}\end{array}\right]^{\mathrm{T}}$.

$$
\boldsymbol{\Lambda}=\mathbf{g}_{B}(\xi)+\mathbf{d}+\mathbf{u}_{B}
$$

The control vector $\mathbf{u}_{B}$ is represented by the product of the control allocation scheme

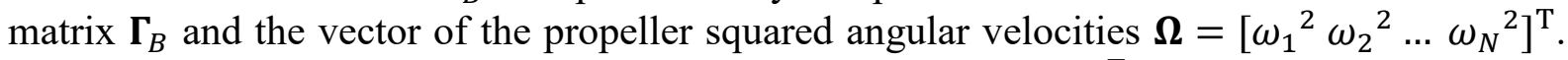
It consists of the rotor system force vector $\mathbf{f}=\left[\begin{array}{lll}f_{X} & f_{Y} & f_{Z}\end{array}\right]^{\mathrm{T}}$ and the moment vector $\boldsymbol{\tau}=\left[\begin{array}{lll}\tau_{\phi} & \tau_{\theta} & \tau_{\psi}\end{array}\right]^{\mathrm{T}}$.

$$
\mathbf{u}_{B}=\Gamma_{B} \boldsymbol{\Omega}
$$

\subsection{Control allocation scheme}

Based on the multirotor configuration and the rotor geometric arrangement, a control allocation scheme can be derived. At first, it is necessary to describe a geometry of connection between the force/moment actuation and the multirotor configuration.

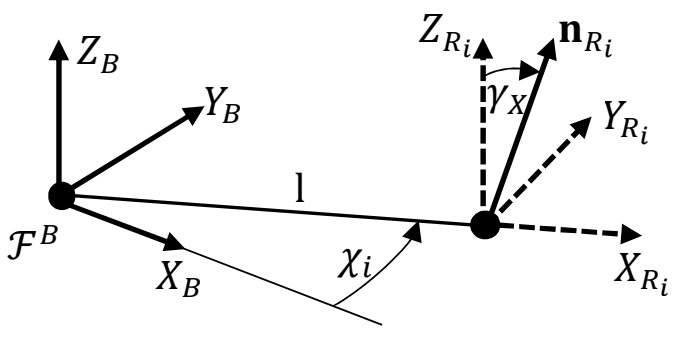

Fig. 3 Rotor coordinate systems

The multirotor configuration consists of an arbitrary number of rotors $(N)$, whereby each rotor propeller generates an aerodynamic force. It is assumed that the aerodynamic force consists of the thrust force and the drag torque. 


\subsubsection{Rotor position and orientation}

Rotor position vector $\xi_{R_{i}}$ is defined as

$$
\xi_{R_{i}}=\left[\begin{array}{c}
\cos \chi_{i} \\
\sin \chi_{i} \\
0
\end{array}\right] \cdot l
$$

where $\chi_{i}$ is the $i$-th rotor angle around the $\mathrm{Z}_{B}$ axis and $l$ is the distance from the rotor to the COG (Fig. 3). The rotor orientation vector of the $i$-th rotor $\mathbf{n}_{R_{i}}$ is defined as:

$$
\mathbf{n}_{R_{i}}=\mathbf{R}\left(\chi_{i}, Z_{B}\right) \mathbf{R}\left(\gamma_{y}, Y_{R_{i}}\right) \mathbf{R}\left(\gamma_{x}, X_{R_{i}}\right) \mathbf{e}_{3}
$$

where $\gamma_{x}$ represents the rotor tilt angle around $X_{R_{i}}$ (the rotor arm axis), $\gamma_{y}$ around the $Y_{R_{i}}$ axis and $\mathbf{e}_{3}$ is a unit vector. If $\gamma_{x}=0$, and $\gamma_{y}=0$, then this is a flat multirotor configuration which means that this is a strongly coupled and underactuated system. If $\gamma_{x} \neq 0$, with proper rotor position and propulsion dynamics it will be shown that it is possible to achieve the desired allocation of the force and the moment in $\left[\begin{array}{llll}\tau_{\phi} & \tau_{\theta} & f_{Z}\end{array}\right]^{\mathrm{T}}$ and $\left[\begin{array}{lll}f_{X} & f_{Y} & \tau_{\psi}\end{array}\right]^{\mathrm{T}}$. By tilting the rotor around $X_{R_{i}}$ it is possible to overcome the underactuation property of the multirotor.

\subsubsection{Propulsion forces and moments}

After the geometry analysis of the multirotor configuration in $\mathcal{F}^{B}$ is presented, the force and moment mapping of the rotor system can be derived. The forces and moments that are generated by the propeller rotation, directly affect the multirotor position and orientation in space. equation

Each propeller generates a force vector which can be calculated by the following

$$
\mathbf{f}_{i}=\left(k_{f} \mathbf{n}_{R_{i}}\right) \omega_{i}^{2}
$$

where $\omega_{i}$ is the angular speed of the $i$-th rotor, and $k_{f}$ is the thrust force factor $\mathrm{Ns}^{2}$.

$$
k_{f}=C_{T} \rho A r^{2}
$$

where $C_{T}$ is the thrust coefficient, $\rho$ is the air density, $A$ is the area of the propeller disk and $r$ is the propeller radius.

Each propeller also generates a moment vector which can be calculated by the following equation

$$
\boldsymbol{\tau}_{i}=\left(k_{f} \xi_{R_{i}} \times \mathbf{n}_{R_{i}}+P_{i} k_{\tau} \mathbf{n}_{R_{i}}\right) \omega_{i}^{2}
$$

where $k_{\tau}$ is the drag torque factor $\mathrm{Nms}^{2}$

$$
k_{\tau}=C_{P} \rho A r^{3}
$$

where $C_{P}$ is the power coefficient.

$P_{i}$ is the signum of the $i$-th propeller rotation. The clockwise $(\mathrm{CW})$ rotation is positive and the counter clockwise (CCW) rotation is negative.

$$
P_{i}=\operatorname{sign}\left(\omega_{i}\right)
$$

The thrust force factor $k_{f}$ and the drag torque factor $k_{\tau}$ can be obtained from experimental measurements. 


\subsubsection{Control allocation scheme matrix}

The control allocation scheme is a $6 \mathrm{xN}$ matrix where 6 represents three forces and three moments while $\mathrm{N}$ represents a number of rotors. It is derived from equations (11) and (13) by using the matrix representation of the vector product $\mathbf{a} \times \mathbf{b}=\mathbf{S}(\mathbf{a}) \mathbf{b}$.

$$
\boldsymbol{\Gamma}_{B}=\left[\begin{array}{ccc}
k_{f} \mathbf{n}_{R_{1}} & \ldots & k_{f} \mathbf{n}_{R_{N}} \\
k_{f} \boldsymbol{S}\left(\boldsymbol{\xi}_{R_{1}}\right) \mathbf{n}_{R_{1}}+P_{1} k_{\tau} \mathbf{n}_{R_{1}} & \ldots & k_{f} \boldsymbol{S}\left(\boldsymbol{\xi}_{R_{N}}\right) \mathbf{n}_{R_{N}}+P_{N} k_{\tau} \mathbf{n}_{R_{N}}
\end{array}\right]
$$

\subsubsection{Control vector}

The rotor system force vector is defined as

$$
\mathbf{f}=\sum_{i=1}^{N} \mathbf{f}_{i}
$$

In the same way, the moment vector is defined as

$$
\boldsymbol{\tau}=\sum_{i=1}^{N} \boldsymbol{\tau}_{i}
$$

Considering the aerodynamic effects, it follows that the control forces and moments are proportional to the squared angular velocities of the propellers. As shown in Fig. 1, the control vector is the input in the rigid body dynamic model. For developing the control design and the control implementation in an aircraft prototype, it is necessary to calculate the angular velocity of each individual rotor.

$$
\boldsymbol{\Omega}=\boldsymbol{\Gamma}_{B}{ }^{-1} \mathbf{u}_{B}
$$

\section{Tilt angle analysis of the passively tilted hexarotor}

Because the tilt angle determines the mapping of rotor angular velocities on the control vector, it is necessary to conduct a tilt angle analysis. We provide an analysis of the hexarotor $\mathrm{X}$ configuration with passively tilted rotors, as shown in Fig. 4.
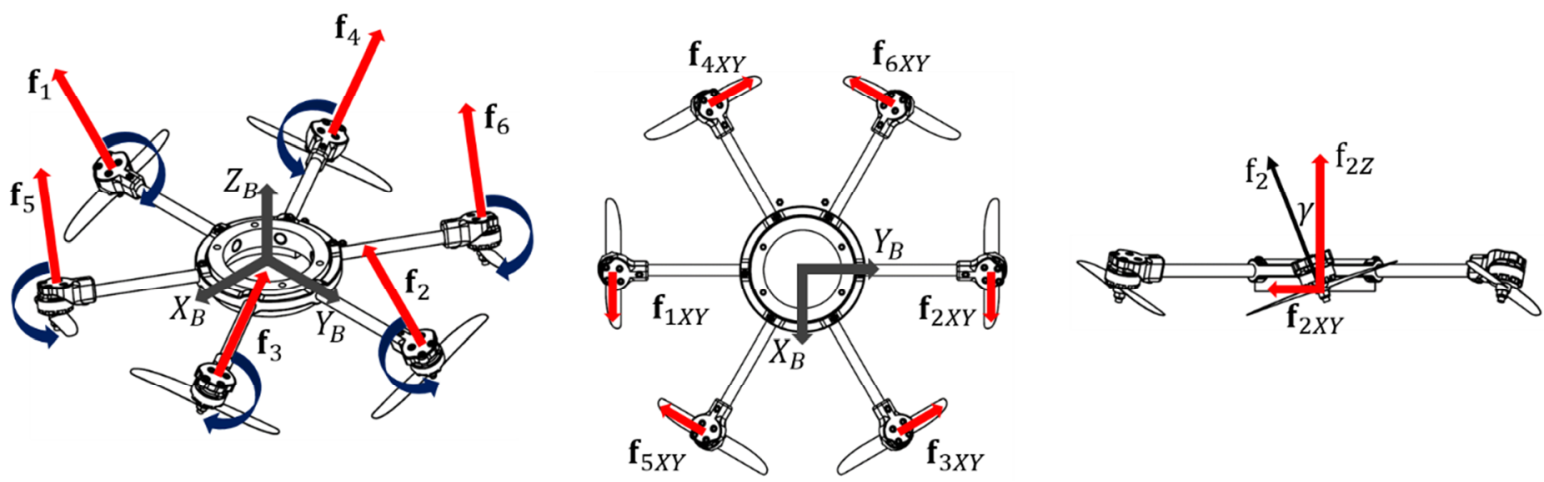

Fig. 4 Fully actuated hexarotor configuration

Clearly, at $\gamma_{x}=0^{\circ}$, there are no forces in the $X_{B} Y_{B}$ plane in $\mathcal{F}^{B}$ and the moment around the $Z_{B}$ axis is relatively small, so there is no possibility to control $f_{X}$ and $f_{Y}$ directly. The control allocation scheme matrix has rank no greater than four.

By increasing the tilt angle, the force along the $Z_{B}$ axis decreases and it is necessary to increase the angular velocities of the rotors in order to keep hover equilibrium. Opposite to the $Z_{B}$ axis, the force along the $X_{B}$ and $Y_{B}$ axes increases. In the presented analysis of the multirotor with six rotors, the control allocation scheme matrix has rank equal to six, indicating that there are six independent controlled DOFs. It means that it is possible to overcome the underactuation property of the multirotor and to decouple orientation from position. It is important to emphasize that for the fully actuated multirotor, it is necessary to 
properly select the orientation of the tilt angles in order to achieve static equilibrium. In our case, all rotors have the same tilt angle and rotors 1, 3 and 6 are tilted $\mathrm{CW}$ around the rotor axis while rotors 2,4 and 5 are tilted CCW.

It can be seen from Fig. 5 that the multirotor maximum available force map values depend on the tilt angles as shown with the force ellipsoids. The maximum available force map values are obtained from the control allocation scheme. By multiplying the map values with squares of angular velocities we get control forces. As can be seen, by increasing the tilt angle, we get control over $f_{X}$ and $f_{Y}$. The considered tilt angle is of up to 30 degrees since a further increase resulted in a significant reduction in the vertical component of the control force.

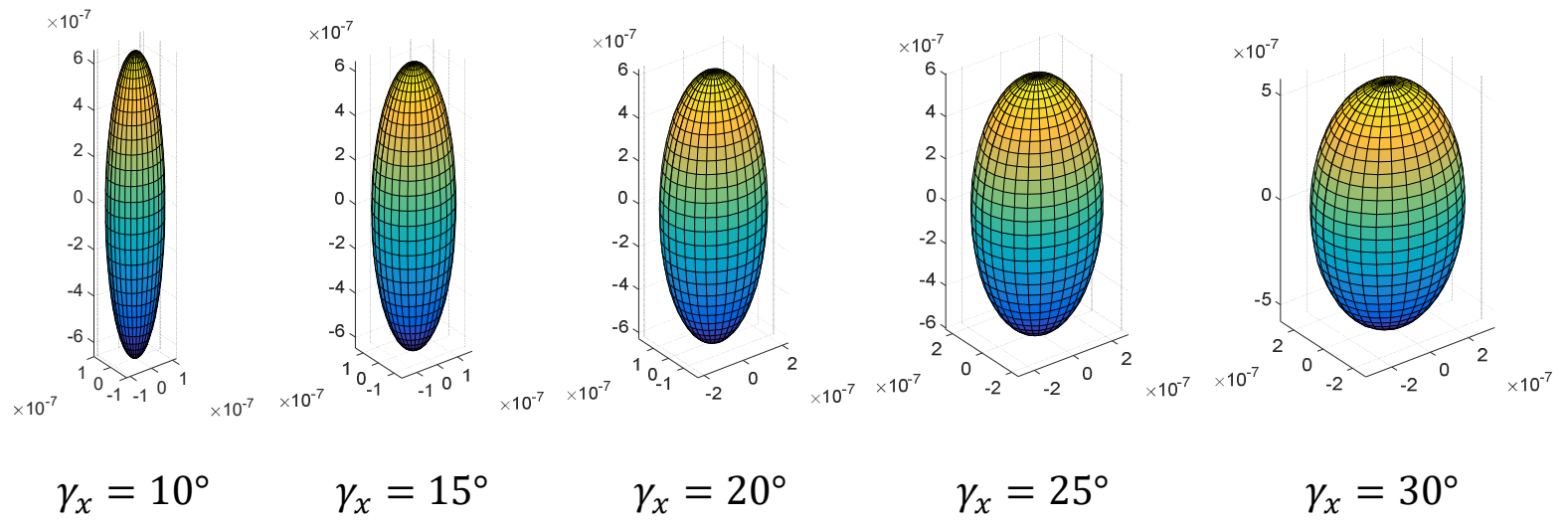

Fig. 5 Force ellipsoids for different tilt angles

Fig. 6 shows the multirotor maximum available moment map values depending on the tilt angle. As the tilt angle increases, the moments $\tau_{x}$ and $\tau_{y}$ slightly decrease, whereas the moment $\tau_{z}$ increases.
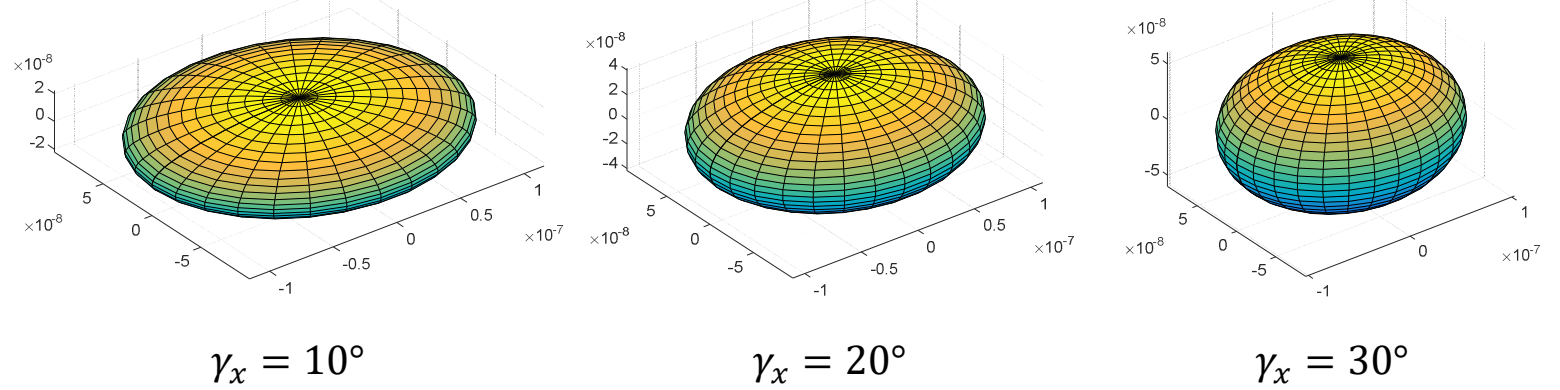

Fig. 6 Moment ellipsoids for different tilt angles

Force maps also depend on the chosen motors and multirotor dimensions. The tilt angle analysis is especially important for a further study of agility, power consumption, component selection, effects of platform size, disturbance rejection, etc.

\section{Tracking control}

After the verification of the mathematical model of a multirotor type of UAVs through a series of open loop simulations, the nonlinear control was developed. Since the control design is based on the fully actuated multirotor UAV it is possible to decouple the attitude and position control. Therefore, the multirotor does not have to tilt as it flies in a horizontal plane, which enables more precise aircraft movements.

Under the assumption that during the multirotor flight, the orientation angles remain near zero [13], the position dynamic model (5) becomes 


$$
\begin{aligned}
& m \ddot{X}=f_{X}+f_{Z} \theta+d_{m X} \\
& m \ddot{Y}=f_{Y}-f_{Z} \phi+d_{m Y} \\
& m \ddot{Z}=-m g+f_{Z}+d_{m Z}
\end{aligned}
$$

And the orientation dynamic model (6) becomes

$$
\begin{aligned}
& I_{X X} \ddot{\phi}=\tau_{\phi}+d_{m \phi} \\
& I_{Y Y} \ddot{\theta}=\tau_{\theta}+d_{m \theta} \\
& I_{Z Z} \ddot{\psi}=\tau_{\psi}+d_{m \psi}
\end{aligned}
$$

which is the fully actuated second order system. External disturbances and modelling errors due to the dynamic model simplification of the original system (7) are represented as the force disturbances $\mathbf{d}_{f}=\left[\begin{array}{lll}d_{m X} & d_{m Y} & d_{m Z}\end{array}\right]^{\mathrm{T}}$ and the moment disturbances $\mathbf{d}_{\tau}=\left[\begin{array}{lll}d_{m \phi} & d_{m \theta} & d_{m \psi}\end{array}\right]^{\mathrm{T}}$.

\subsection{Control structure of the fully actuated multirotor}

The control goal is the asymptotic tracking of the reference trajectory $\xi_{\text {des }}(t)=\left[\begin{array}{lll}X_{\text {des }}(t) & Y_{\text {des }}(t) & Z_{\text {des }}(t)\end{array}\right]^{\mathrm{T}}$ under preserving the orientation angles at zero, in the presence of unknown external disturbances.

The tracking error variables are

$$
\tilde{\xi}=\xi-\xi_{\text {des }}(t)
$$

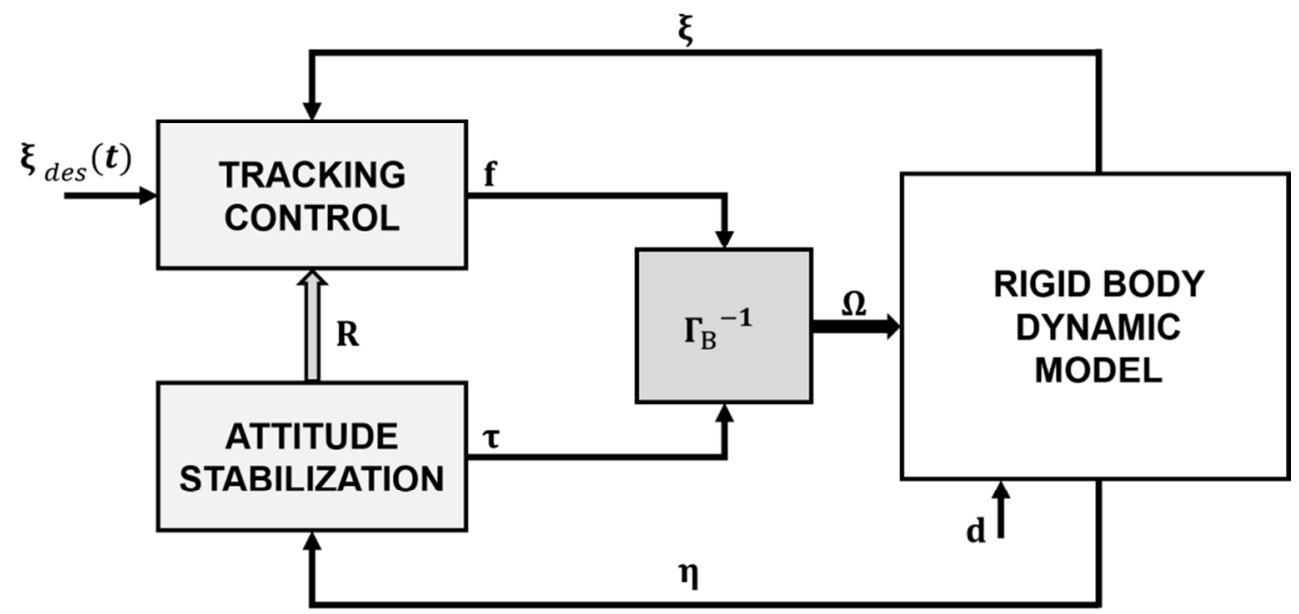

Fig. 7 Fully actuated multirotor control scheme

\subsection{Sliding-mode control (SMC) design}

The controller compensates for the unmodelled dynamics and external disturbances by using the sliding-mode control laws. It is divided into the tracking control, which is basically a position controller, and the attitude (orientation) controller.

\subsubsection{Position controller} errors $\tilde{\xi}$.

The first step is to design the control law for $\mathbf{f}$ which will stabilize the position tracking 


$$
m \ddot{\widetilde{\xi}}=-m \ddot{\boldsymbol{\xi}}_{\text {des }}-m \mathbf{g}_{B}(\boldsymbol{\xi})+\mathbf{f}+\mathbf{d}_{f}
$$

The control variable force vector is chosen as

$$
\mathbf{f}=m \mathbf{g}_{B}(\boldsymbol{\xi})-\mathbf{K}_{D} \mathbf{s}_{f}-\boldsymbol{\rho}_{f} \operatorname{sign}\left(\mathbf{s}_{f}\right)
$$

where $\mathbf{K}_{D}$ is a constant positive definite diagonal gain matrix. $\mathbf{s}_{f}=\left[\begin{array}{lll}S_{X} & S_{Y} & s_{Z}\end{array}\right]^{\mathrm{T}}$ is a vector of sliding variables and it has the following form

$$
\mathbf{s}_{f}=\dot{\tilde{\xi}}+\alpha \tilde{\xi}
$$

where $\alpha$ is a positive parameter. The diagonal matrix $\boldsymbol{\rho}_{f}=\operatorname{diag}\left\{\rho_{X}, \rho_{Y}, \rho_{Z}\right\}$ is defined by using the Lyapunov stability analysis [13].

\subsubsection{Attitude controller}

The aim of the attitude controller is to keep the multirotor orientation angles equal to zero since the purpose of the controller is to achieve aircraft movements without the need to tilt.

$$
\mathbf{I} \ddot{\eta}=\boldsymbol{\tau}+\mathbf{d}_{\tau}
$$

The control variable moment vector is chosen as

$$
\boldsymbol{\tau}=-\mathbf{K}_{D} \mathbf{s}_{\tau}-\boldsymbol{\rho}_{\tau} \operatorname{sign}\left(\mathbf{s}_{\tau}\right)
$$

where $\mathbf{S}_{\tau}=\left[\begin{array}{lll}S_{\phi} & S_{\theta} & S_{\psi}\end{array}\right]^{\mathrm{T}}$ is the vector of sliding variables and it has the following form

$$
\mathbf{s}_{\tau}=\dot{\boldsymbol{\eta}}+\alpha \boldsymbol{\eta}
$$

The diagonal matrix $\boldsymbol{\rho}_{\tau}=\operatorname{diag}\left\{\rho_{\phi}, \rho_{\theta}, \rho_{\psi}\right\}$ is defined by using the Lyapunov stability analysis [13].

The proposed control design provides asymptotic tracking of the reference trajectory with external disturbance rejection. Although the control goal is satisfied, the discontinuous controller suffers from the chattering phenomenon. Therefore, the RISE control design is introduced which eliminates the control variable chattering.

\subsubsection{Comparison between SMC designs of flat and of non-flat multirotor configurations}

By comparing the presented controller in the non-flat multirotor configuration (NFMRC) with the controller in the flat multirotor configuration (FMRC) presented in [13], several advantages of the NFMRC should be emphasized. In the case of the NFMRC, the closed-loop error dynamics is of the second order for each DOF, so that only the position and velocity measurements are needed for the controller implementation. In the case of the FMRC, the closed-loop error dynamics for the positions $X$ and $Y$ are of the fourth order, so that additional filters for the estimation of the second and third position derivatives are necessary. Such a controller is more demanding for implementation and conditions for the closed-loop stability are more complex.

Further, the SMC gains in the case of the NFMRC depend only on the maximum amplitude of external disturbances, while in the case of the FMRC, the SMC gains depend also on the second derivatives of external disturbances in the horizontal X-Y plane. In other words, the SMC in the FMRC is more sensitive to wind gusts in the horizontal plane, even in the case of small amplitudes. 


\subsection{RISE control design}

Chattering can be described as high-frequency oscillations of the actuator dynamics which results in actuator failure in a real system. To overcome the chattering problem of control variables and preserve the tracking properties of the sliding mode controller, a RISE control design is proposed.

The control variable is chosen as

$$
\mathbf{f}=m \mathbf{g}_{B}(\xi)-\mathbf{K}_{D} \mathbf{s}_{f}+\mathbf{Y}
$$

where $\mathbf{Y}=\left[\begin{array}{lll}v_{X} & v_{Y} & v_{Z}\end{array}\right]^{\mathrm{T}}$ is a vector of RISE controller variables. Its derivative has the following form

$$
\dot{\boldsymbol{Y}}=-\mathbf{K}_{I} \mathbf{s}_{f}-\boldsymbol{\rho}_{I} \operatorname{sign}\left(\mathbf{s}_{f}\right)
$$

where $\boldsymbol{\rho}_{I}=\operatorname{diag}\left\{\rho_{I}, \rho_{I}, \rho_{I}\right\}$, and $\mathbf{K}_{I}$ is the constant positive definite diagonal gain matrix. With the proposed controller, high-frequency switching of the control variables is avoided, since the chattering caused by the discontinuous signum function is filtered by the integrators in (30).

The positive definite gain matrices $\mathbf{K}_{D}, \mathbf{K}_{I}, \boldsymbol{\rho}_{I}$ and the positive parameter $\alpha$ satisfy the stability conditions which are adopted from [14] for the case of constant gravitational forces

$$
\begin{aligned}
& \lambda_{m}\left\{\mathbf{K}_{D}\right\}>2 \alpha \bar{m} \\
& \lambda_{m}\left\{\boldsymbol{\rho}_{I}\right\}>\bar{m}\left\|\dddot{\boldsymbol{\xi}}_{\text {des }}\right\|_{1 M}+\left\|\dot{\mathbf{d}}_{f}\right\|_{1 M}+\frac{1}{\alpha}\left(\bar{m}\left\|\xi_{\text {des }}^{(4)}\right\|_{1 M}+\left\|\ddot{\mathbf{d}}_{f}\right\|_{1 M}\right)
\end{aligned}
$$

where the notation $\lambda_{m}\{$.$\} represents the minimal matrix eigenvalue, \|\mathbf{x}(t)\|_{1 M}=$ $\max _{t} \sum_{i=1}^{n}\left|x_{i}(t)\right|$ for some vector $\mathbf{x}(t)$, and $\bar{m}>m$ is the upper estimation of the multirotor mass $m$.

\section{Simulation results}

The system is tested in numerical simulations by using the $4^{\text {th }}$ order Runge-Kutta method with a fixed time step.

The physical parameters of the rigid body are:

$$
I_{X X}=0.0049 \mathrm{Nms}^{2}, I_{Y Y}=0.0058 \mathrm{Nms}^{2}, I_{Z Z}=0.0095 \mathrm{Nms}^{2}, m=1.2 \mathrm{~kg}, g=9.81 \mathrm{~m} / \mathrm{s}^{2} \text {. }
$$

The physical parameters of the rotor dynamics and geometry of the passively tilted hexarotor $\mathrm{X}$ multirotor configuration are:

$$
k_{f}=6.7 \times 10^{-7} \mathrm{Ns}^{2}, \quad k_{\tau}=1.8 \times 10^{-9} \mathrm{Nms}^{2}, \quad l=0.175 \mathrm{~m}, \quad \gamma_{x}=20^{\circ}, \quad \gamma_{y}=0 .
$$

The reference trajectory chosen for $\boldsymbol{\xi}_{\text {des }}(t)$ is

$$
X_{\text {des }}(t)=\cos (0.5 t), \quad Y_{\text {des }}(t)=\sin (0.5 t), \quad Z_{\text {des }}(t)=0.5 t
$$

The initial conditions are: $X(0)=0.5, Y(0)=0, Z(0)=0, \phi(0)=-10^{\circ}, \theta(0)=10^{\circ}$ and $\psi(0)=60^{\circ}$. All other initial conditions are set to zero. 
External disturbances have been introduced, such as wind gusts, which affect the control force vector in different time samples, which can be modelled by the forces $d_{m X}, d_{m Y}$ and $d_{m Z}$.

$$
\begin{aligned}
& d_{m X}=e^{-\left(t-\frac{T}{4}\right)^{2}} \\
& d_{m Y}=e^{-\left(t-\frac{T}{2}\right)^{2}} \\
& d_{m Z}=e^{-\left(t-\frac{3 T}{4}\right)^{2}}
\end{aligned}
$$

Where $T$ is the total simulation time.

The SMC gains for $f_{X}, f_{Y}, f_{Z}, \tau_{\phi}, \tau_{\theta}$ and $\tau_{\psi}$ are chosen as: $\mathbf{K}_{D}=\operatorname{diag}\left\{\begin{array}{lll}5 & 5 & 5\end{array}\right\}$, $\alpha=1.5$ and $\rho_{X}=\rho_{Y}=\rho_{Z}=\rho_{\phi}=\rho_{\theta}=\rho_{\psi}=10$. The controller gains are chosen in accordance with the stability conditions (31) and (32): $\mathbf{K}_{I}=\operatorname{diag}\left\{\begin{array}{llll}2.5 & 2.5 & 2.5\end{array}\right\}$ and $\rho_{I}=2.5$.

In Figures 8-10, we can see simulation results for the SMC of a fully actuated multirotor in the case when external disturbances are introduced. The proposed controller provides exponential tracking of the reference trajectory while preserving the orientation angles equal to zero, which can be seen in Fig. 8.
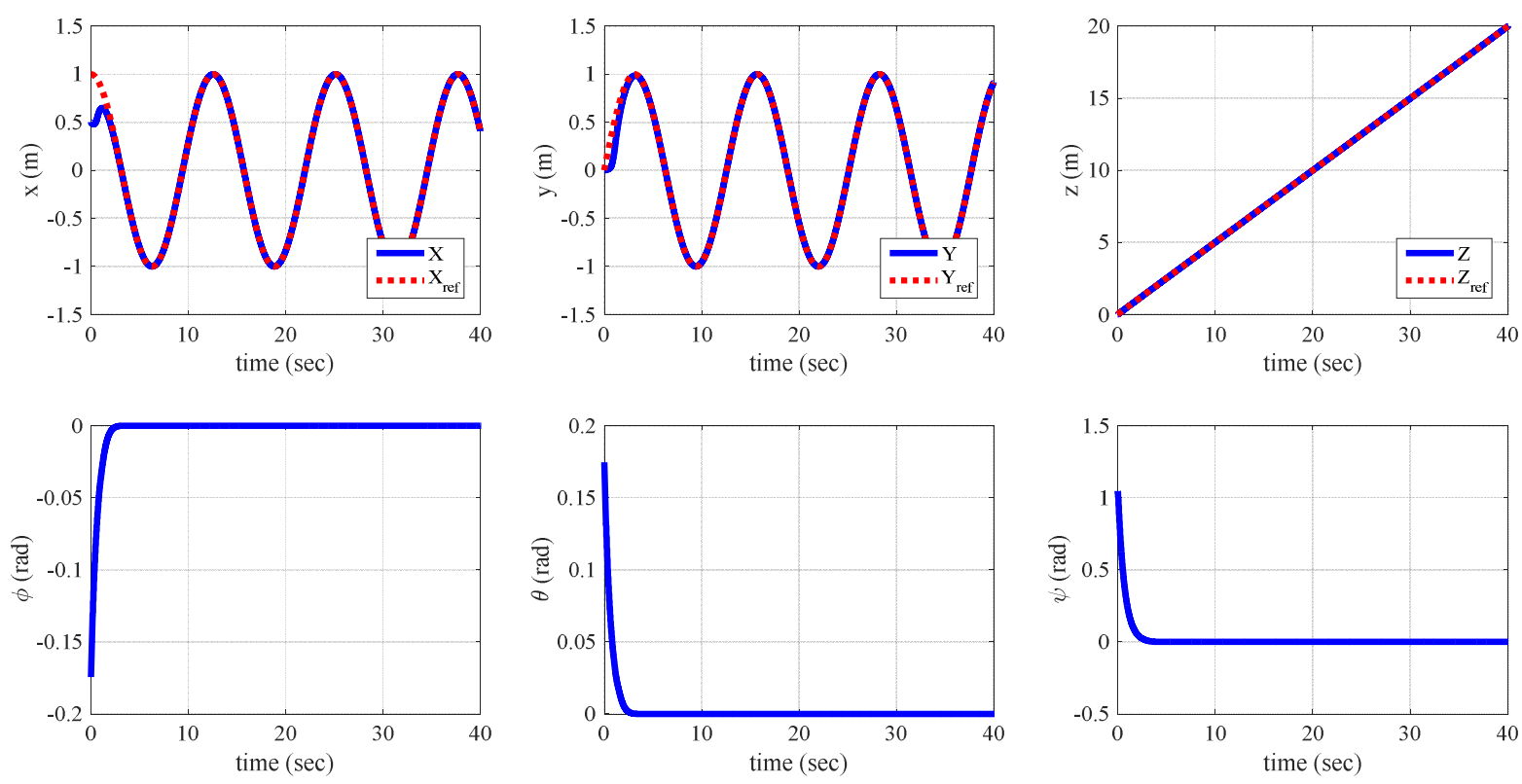

Fig. 8 Position and orientation of multirotor in closed-loop SMC in the presence of external disturbances
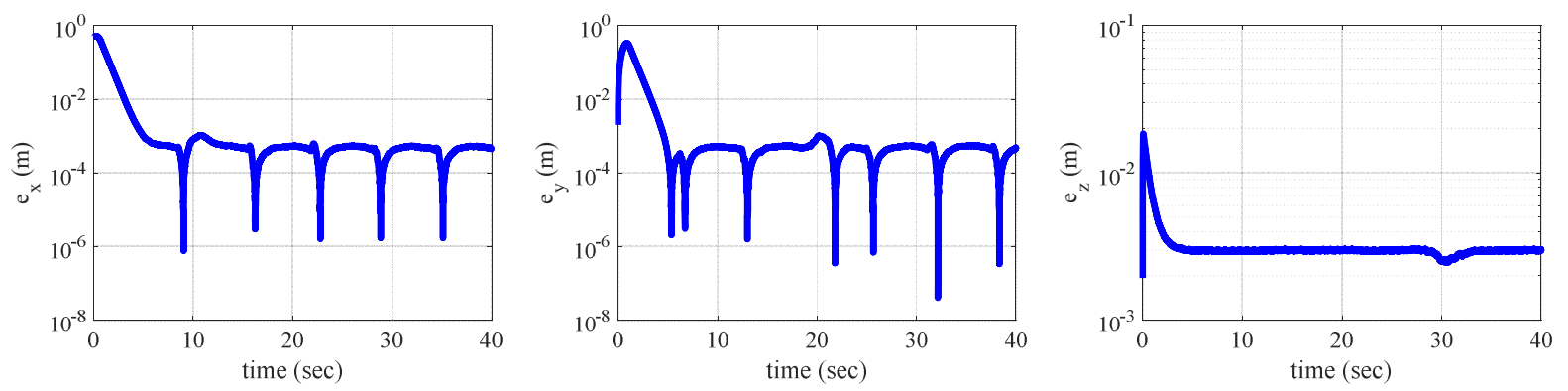

Fig. 9 Position errors of multirotor in closed-loop SMC in the presence of external disturbances 


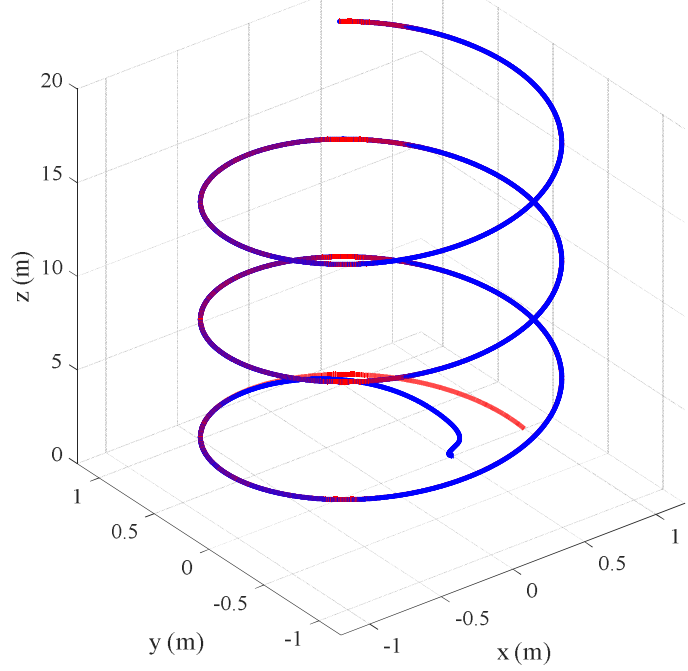

Fig. 10 The multirotor and reference - SMC

Although the SMC provides asymptotic tracking of the reference trajectory, highfrequency oscillations of control variables occur. In Fig. 11 we can see control forces for the $\mathrm{SMC}$ in the presence of external disturbances, which are shown in Fig. 12.
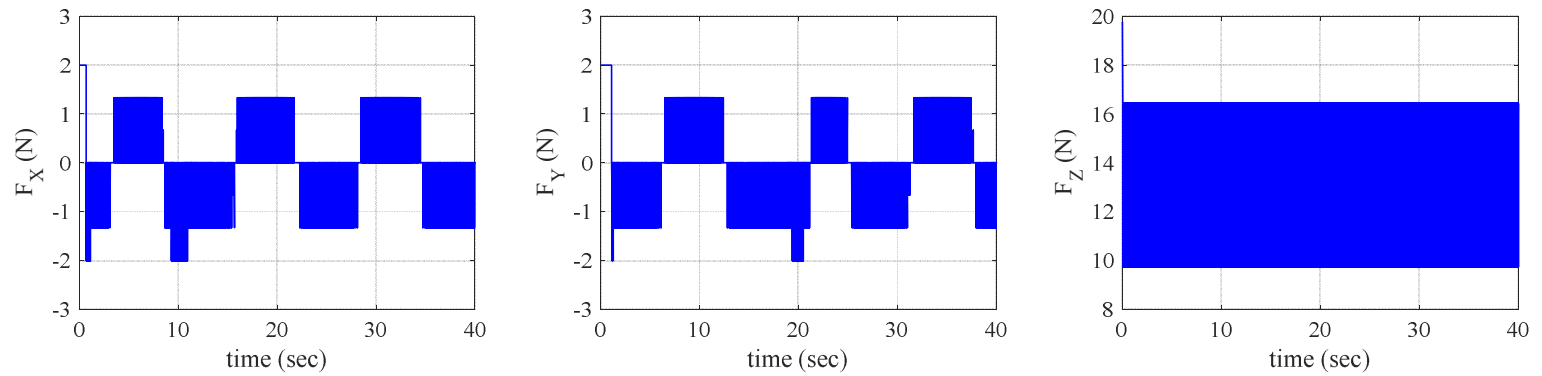

Fig. 11 Forces of multirotor in closed-loop with SMC control in the presence of external disturbances
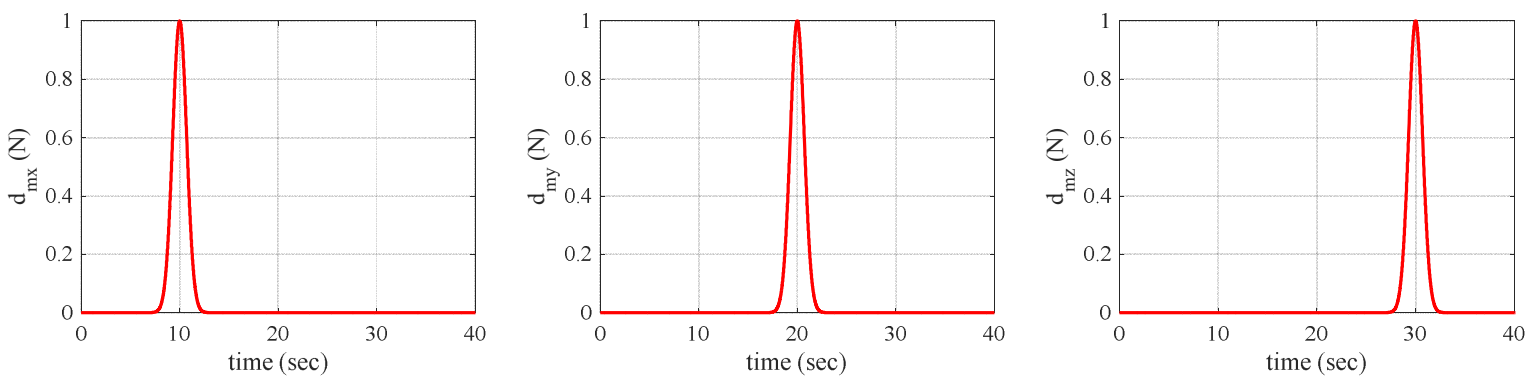

Fig. 12 External force disturbances

Figures 13-15 show simulation results for the proposed RISE tracking controller in the case when external disturbances are present.
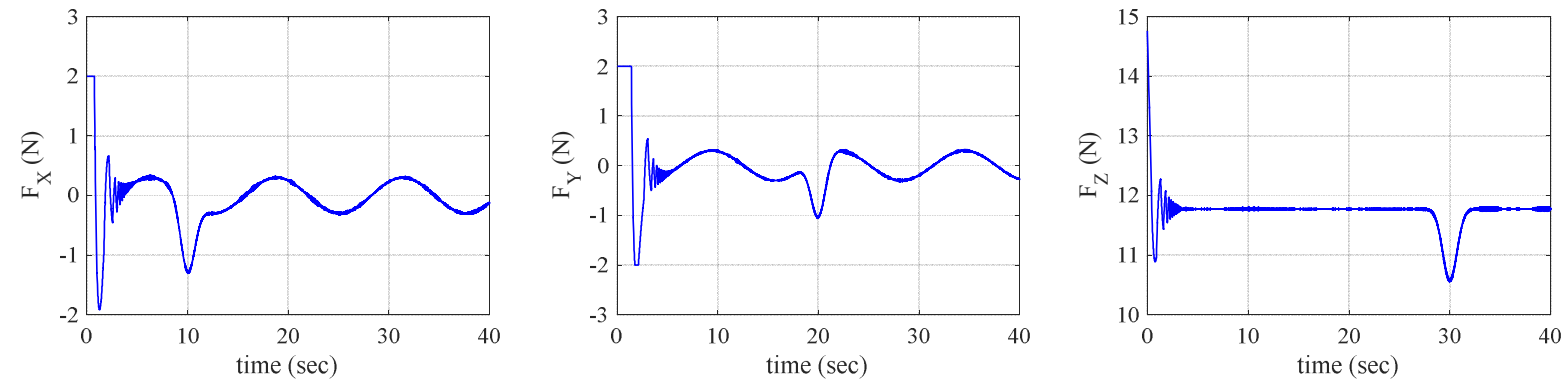

Fig. 13 Forces of multirotor in closed-loop with RISE control in the presence of external disturbances 


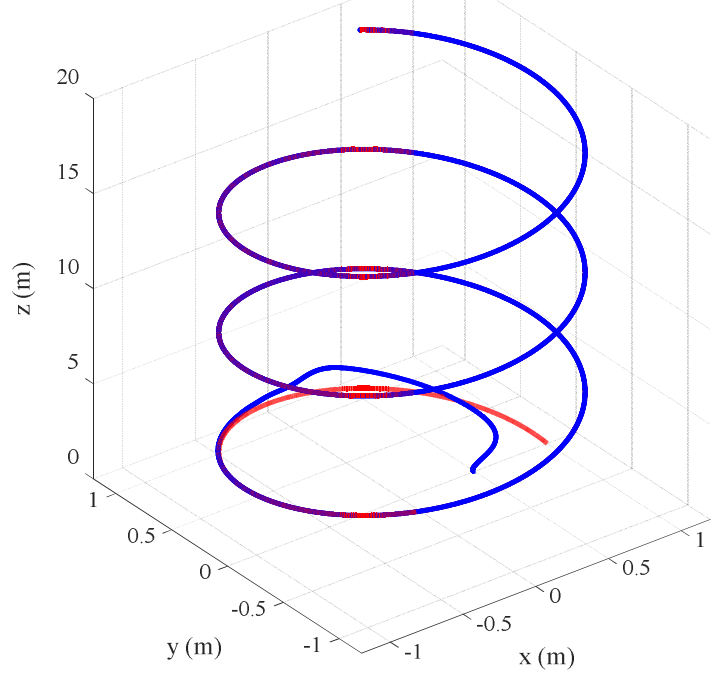

Fig. 14 Forces of multirotor in closed-loop with RISE control in the presence of external disturbances
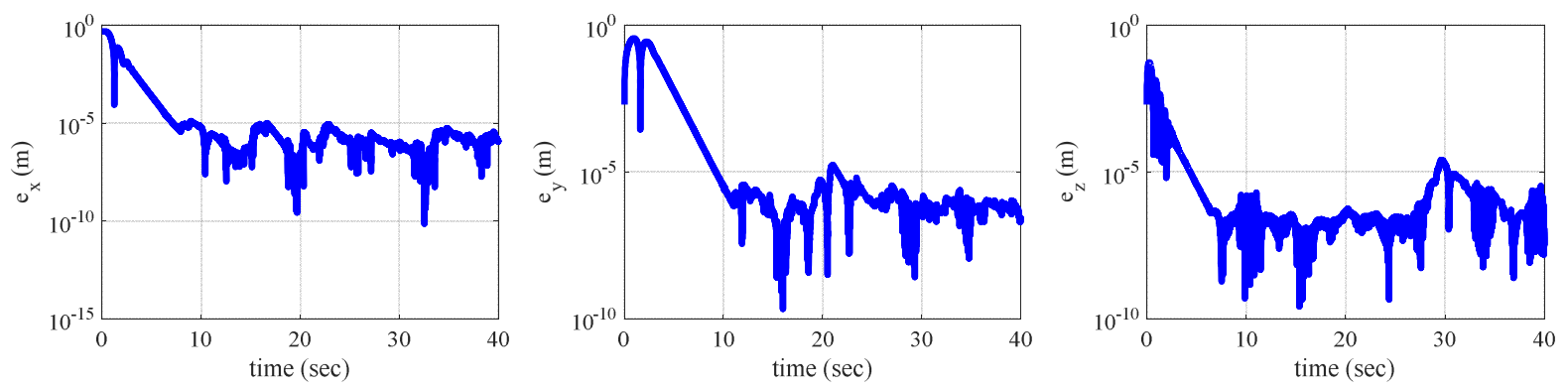

Fig. 15 Position errors of multirotor in closed-loop RISE control in the presence of external disturbances

In Fig. 13, the cancellation of the chattering phenomenon can be seen. The RISE control design shows satisfying results for asymptotic tracking in the case when external disturbances on the multirotor occur. In addition, the RISE control decreases the magnitude of error as shown in Fig. 15.

\section{Conclusion}

In this paper, a control allocation scheme for a multirotor UAV is presented. It describes a mapping of the rotor angular velocities to the control vector, which is necessary for the controller implementation in a multirotor system. It also enables an analysis of various multirotor UAV properties in order to design a multirotor configuration for different purposes. By analysing the force and moment mapping it is possible to design multirotor configurations which overcome the underactuation property of flat multirotor configurations. Configurations with six or more passively tilted rotors can achieve full controllability, therefore it is possible to decouple position from orientation. The control design is based on the fully actuated multirotor UAV with a decoupled position and attitude control. SMC control laws were considered for robust tracking of the reference trajectory in the presence of external disturbances. The simulations have shown that the control goal is satisfied but it is necessary to eliminate the chattering phenomenon of control variables. For this purpose, a RISE controller is proposed for a fully actuated multirotor with passively tilted rotors. The presented simulation results shown that the proposed RISE controller provides cancellation of chattering for the case of asymptotic tracking in the presence of external disturbances on the multirotor.

Future work will focus on the multirotor configuration design based on identified and estimated physical parameters of the rotor system. The designed prototype will be used for the implementation of the control allocation scheme and the tracking control algorithms. 


\section{REFERENCES}

[1] Kumar, V., Michael, N. (2012). Opportunities and challenges with autonomous micro aerial vehicles, The International Journal of Robotics Research, Vol. 31(11), 1279-1291. https://doi.org/10.1177/0278364912455954

[2] Mulgaonkar, Y., Whitzer, M., Morgan, B., Kroninger, C. M., Harrington, A. M., Kumar, V. (2014). Power and Weight Considerations in Small, Agile, Quadrotors, Proceedings of SPIE - The International Society for Optical Engineering, Vol 9083, Baltimore, Maryland, USA

[3] Otsuka, H., Nagatani, K. (2016). Thrust Loss Saving Design of Overlapping Rotor Arrangement on Small Multirotor Unmanned Aerial Vehicles, 2016 IEEE International Conference on Robotics and Automation (ICRA), 3242-3248, Stockholm, Sweden. https://doi.org/10.1109/ICRA.2016.7487494

[4] Theys, B., Dimitriadis, G., Hendrick, P., De Schutter, J. (2016) Influence of propeller configuration on propulsion system efficiency of multi-rotor Unmanned Aerial Vehicles, 2016 International Conference on Unmanned Aircraft Systems (ICUAS), 195-201, Arlington, USA. https://doi.org/10.1109/ICUAS.2016.7502520

[5] Mizutani, S., Okada, Y., Salaan, C. J., Ishii, T., Ohno, K., Tadokoro, S. (2015) Proposal and Experimental Validation of a Design Strategy for a UAV with a Passive Rotating Spherical Shell, 2015 IEEE/RSJ International Conference on Intelligent Robots and Systems (IROS) 1271-1278, Hamburg, Germany.

[6] Briod, A., Kornatowski, P., Zufferey, J. C., Floreano, D. (2014) A Collision-resilient Flying Robot, Journal of Field Robotics, Vol 31(4), 496-509. https://doi.org/10.1002/rob.21495

[7] Driessens, S., Pounds, P. (2015) The Triangular Quadrotor: A More Efficient Quadrotor Configuration, IEEE Transactions on Robotics, Vol 31, No. 6, 1517-1526. https://doi.org/10.1109/TRO.2015.2479877

[8] Badr, S., Mehrez, O., Kabeel, A. E. (2016) A Novel Modification for a Quadrotor Design, 2016 International Conference on Unmanned Aircraft Systems (ICUAS), 702-710, Arlington, USA. https://doi.org/10.1109/ICUAS.2016.7502536

[9] Ryll, M., Bulthoff, H. H., Giordano, P. R. (2014) A Novel Overactuated Quadrotor Unmanned Aerial Vehicle: Modeling, Control, and Experimental Validation, IEEE Transactions on Control Systems Technology, Vol 23, No 2, 540-556. https://doi.org/10.1109/TCST.2014.2330999

[10] Jiang, G., Voyles, R. (2014) A Nonparallel Hexrotor UAV with Faster Response to Disturbances for Precision Position Keeping, 2014 IEEE International Symposium on Safety, Security, and Rescue Robotics (SSRR), Toyako-cho, Hokkaido, Japan.

[11] Cabecinhas, D., Cunha, R., Silvestre, C. (2015), A Globally Stabilizing Path Following Controller for Rotorcraft With Wind Disturbance Rejection, IEEE Transactions on Control Systems Technology, Vol 23(2), 708-714. https://doi.org/10.1109/TCST.2014.2326820

[12] Wang L., Su, J. (2015) Robust Disturbance Rejection Control for Attitude Tracking of an Aircraft, IEEE Transactions on Control Systems Technology, Vol 23(6). https://doi.org/10.1109/TCST.2015.2398811

[13] Kasać, J., Stevanović, S., Žilić, T., Stepanić, J. (2013) Robust Output Tracking Control of a Quadrotor in the Presence of External Disturbances, Transactions of FAMENA, Vol 37(4), pp. 29-42.

[14] Kasać, J., Žilić, T., Milić, V., Jokić A., Lobrović M. (2016) Robust Decentralized Global Asymptotic Tracking Control of a Class of Nonlinear Mechanical Systems, American Control Conference (ACC), Boston, MA, USA.

[15] Cai, G., Chen, B. M., Lee, T. H. (2011) Unmanned Rotorcraft Systems, Springer-Verlag, London. https://doi.org/10.1007/978-0-85729-635-1

[16] Fossen, T. I. (1994). Guidance and Control of Ocean Vehicles, John Wiley \& Sons, New York.

[17] Bresciani, T. (2008) Identification and control of a quadrotor helicopter, Master Thesis, Department of Automatic Control, Lund University.

Submitted: $\quad 15.12 .2016$

Accepted: $\quad$ 18.7.2017
Denis Kotarski, denis.kotarski@vuka.hr Karlovac University of Applied Sciences, Trg J.J.Strossmayera 9, Karlovac, Croatia Petar Piljek, petar.piljek@fsb.hr Hrvoje Brezak, hb181313@fsb.hr Josip Kasać, josip.kasac@fsb.hr Faculty of Mechanical Engineering and Naval Architecture, Ivana Lučića 5,10002 Zagreb, Croatia 